\title{
Relação entre desvios fonológicos e processamento auditivo
}

\section{Relationship between phonological disorders and auditory processing}

\author{
Débora Tomazi Moreira Caumo', Maria Inês Dornelles da Costa Ferreira²
}

\begin{abstract}
RESUMO
Objetivos: Pesquisar a relação entre desvio fonológico e processamento auditivo. Métodos: Os dados foram coletados por meio da verificação de prontuários. Foram incluídos no estudo pacientes com diagnóstico de desvio fonológico que realizaram testes de processamento auditivo e que tinham idade mínima de sete anos. Considerou-se a avaliação do processamento auditivo, a avaliação da fala, o gênero, a idade e a série escolar. Resultados: Todas as crianças (100\%) apresentaram pelo menos um subperfil do processamento auditivo alterado. Ao comparar os processos de substituição e de estruturação silábica aos resultados dos testes de processamento auditivo verificou-se correlação estatisticamente significante para a etapa de integração binaural para a orelha direita do teste dicótico de dígitos $(\mathrm{p}=0,018)$ e para a condição nomeando do teste PPS $(\mathrm{p}=0,041)$. Na comparação dos testes de processamento auditivo com a idade encontrou-se diferença estatisticamente significante para o teste PSI na orelha direita ( $\mathrm{p}=0,011)$ para a faixa de 10 a 12 anos. O mesmo ocorreu na comparação com a série escolar, em que o teste SSW na condição direita competitiva ( $\mathrm{p}=0,039)$ e a atenção direcionada à direita do teste dicótico de dígitos $(\mathrm{p}=0,037)$ foram estatisticamente significantes para as séries mais avançadas. Conclusão: A pesquisa sugere a existência de uma estreita relação entre processamento auditivo e desvio fonológico principalmente em relação ao desempenho da orelha direita, evidenciando a importância de determinar a existência do comprometimento das habilidades auditivas em crianças com desvio fonológico.
\end{abstract}

Descritores: Audição; Percepção auditiva; Deficiências do desenvolvimento; Linguagem; Distúrbios da fala

\section{INTRODUÇÃO}

Para refletir sobre a relação entre desvio fonológico e processamento auditivo torna-se necessário o estudo de alguns tópicos referentes a esses assuntos.

O desvio fonológico ocorre quando a fala de uma criança é caracterizada por omissões e substituições de fonemas. Essa dificuldade refere-se à organização e classificação dos sons que ocorrem contrastivamente na língua, ou seja, são dificuldades de organização mental, de estabelecimento do sistema da língua-alvo e de adequação à informação oral recebida ${ }^{(1)}$. Também é possível concordar com outros autores que classificam os transtornos fonológicos em cinco subtipos: atraso de fala genético, otite média com efusão, apraxia de desenvolvimento, envolvimento psicológico e erros residuais ${ }^{(2)}$.

$\mathrm{Na}$ aquisição fonológica normal, o domínio do sistema fonológico da língua alvo é atingido espontaneamente, em

Trabalho de conclusão do Curso de Fonoaudiologia realizado no Laboratório de Audiologia do Centro Universitário Metodista IPA - Porto Alegre (RS), Brasil.

(1) Fonoaudióloga clínica - Porto Alegre (RS), Brasil.

(2) Doutora, Professora dos Cursos de Fonoaudiologia do Centro Universitário Metodista IPA - Porto Alegre (RS), Brasil e da Faculdade Nossa Senhora de Fátima - Caxias do Sul (RS), Brasil.

Endereço para correspondência: Maria Inês Dornelles da Costa Ferreira. R. Luiz Afonso, 158/702, Cidade Baixa, Porto Alegre - RS, CEP: 90050-310.

E-mail: costa.ferreira@terra.com.br

Recebido em: 17/3/2008; Aceito em: 29/7/2008 uma sequência e faixa etária comum à maior parte das crianças (quatro a seis anos). A aquisição fonológica com desvios fonológicos, por outro lado, é aquela na qual a adequação do sistema fonológico da língua alvo não é atingida espontaneamente e/ou na mesma sequência constatada na maioria das crianças, nem ocorre na faixa etária mencionada. Dessa forma, o desvio fonológico envolve o componente fonológico da linguagem, caracterizando-se por dificuldades na organização mental dos sons. Sua etiologia é desconhecida, embora alguns trabalhos apontem fatores influentes ${ }^{(3)}$.

Assim, o desvio fonológico é um evento que pode ser identificado por meio de processos fonológicos que ocorrem na fala das crianças em função das simplificações das regras fonológicas da língua. Ou seja, a maioria dos processos fonológicos tende a simplificar a forma adulta de falar pelo apagamento de sons ou pela substituição dos mesmos.

Assim, os processos fonológicos podem ser classificados em processos de estruturação silábica e de substituição. Os processos de estrutura silábica ocorrem quando a forma silábica de uma palavra alvo é alterada. Envolve apagamento de líquida (inicial, intervocálica e final), apagamento de fricativa final, redução de encontro consonantal, apagamento de sílaba átona, metátese (reordenação de sons dentro da mesma palavra) e epêntese (inserção de uma vogal entre duas consoantes). Já, os processos de substituição envolvem: anteriorização, posteriorização, plosivização, substituição de líquidas, semivocalização de líquida, assimilação, sonorização pré-vocálica, dessonorização 
ou outras formas de substituições ${ }^{(4)}$. A referida classificação foi utilizada neste estudo, embora outros trabalhos realizados no estado de São Paulo apresentam outra forma de classificação dos processos fonológicos: simplificação do encontro consonantal, simplificação de líquidas, eliminação da consoante final, ensurdecimento de fricativa, ensurdecimento de plosiva, frontalização da palatal, simplificação da velar, posteriorização para palatal, frontalização da velar e plosivização de fricativa ${ }^{(5)}$.

As habilidades de linguagem e fala dependem da integridade e maturação neuromuscular e do sistema sensorial. No aprendizado de um padrão articulatório de uma língua estão envolvidos aspectos sensoriais e motores, sendo um desses aspectos sensoriais, as habilidades auditivas relacionadas à percepção da fala ${ }^{(6)}$. As privações sensoriais causadas ou por ambientes privados culturalmente, ou por perdas auditivas, mesmo as temporárias causadas por otite média, podem criar desvantagens para a aquisição e desenvolvimento, tanto da produção de fala como da linguagem de uma criança ${ }^{(6-7)}$.

Alguns autores afirmam que para o desenvolvimento da fala e da linguagem é fundamental a habilidade de analisar e interpretar os estímulos sonoros, como localizar e memorizar sons. Tais habilidades ocorrem a partir do processamento auditivo da informação definido como a capacidade de organizar e compreender os estímulos sonoros e envolve um conjunto de habilidades necessárias para atender, discriminar, reconhecer, armazenar e compreender a informação auditiva. Também se refere ao caminho percorrido pelo estímulo acústico, desde sua entrada no sistema auditivo até o córtex ${ }^{(8)}$.

$\mathrm{Na}$ avaliação auditiva central, a faixa etária deve ser considerada, já que o desempenho do indivíduo nos testes de processamento auditivo aumenta com o avanço da idade até a pré-adolescência com pouca variação na idade adulta. São sugeridos para a faixa etária de três anos e seis meses a quatro anos e seis meses os testes de localização sonora em cinco direções, memória sequencial verbal e não verbal com três objetos (sino, guizo e agogô) $)^{(9)}$ e teste de frases com mensagem competitiva ipsilateral (Pediatric Speech Intelligibility - PSI). Para crianças com idade entre quatro anos e seis meses e cinco anos podem ser realizados os testes de localização, memória sequencial verbal e não verbal com três objetos, PSI com frases e teste dicótico com sons não verbais. De seis a sete anos, sugere-se os testes de localização, de memória sequencial verbal e não verbal com quatro objetos (sino, guizo, agogô e côco $)^{(9)}$, PSI, fala filtrada e fusão binaural, fala com ruído e dicótico de dígitos. De oito anos em diante podem ser realizados todos os testes disponíveis memória sequencial verbal e não verbal, localização sonora, PSI, dicótico de dígitos, SSW, fala filtrada, fusão binaural, PPS, DPS, RGDT e GIN.

O diagnóstico dos transtornos de processamento auditivo é atribuído a um conjunto de testes comportamentais e/ou eletrofisiológicos que classificam as habilidades auditivas em subperfis.

Os subperfis específicos do processamento auditivo são decodificação, integração e função não-verbal. Já os subperfis denominados associação e organização de saída são considerados subperfis secundários ao processamento auditivo por envolver outras habilidades como atenção, memória e funções executivas $^{(10)}$.
A decodificação auditiva é o subperfil mais específico à modalidade auditiva. Este pode ser identificado por alterações nas habilidades auditivas de fechamento, discriminação auditiva, processamento temporal, separação e integração binaural e, ainda, localização sonora. Indivíduos com alteração neste subperfil podem apresentar fadiga auditiva e quebra da informação no nível fonêmico. Os achados típicos na avaliação comportamental do processamento auditivo são: déficit bilateral ou na orelha direita nos testes de escuta dicótica, desempenho rebaixado em testes monoaurais de baixa redundância e de localização sonora ${ }^{(10-11)}$.

Disfunções em prosódia ou função não verbal caracterizamse por inabilidade em compreender e/ou identificar intenções de um enunciado, dificuldade em entender piadas e mensagens com duplo sentido e dificuldades em expressar o pensamento. Referem-se aos aspectos supra-segmentais da linguagem. Os achados na avaliação comportamental do processamento auditivo são tipicamente: déficit na nomeação e na imitação de padrões temporais nos testes PPS (Pich Pattern Sequence) e DPS (Duration Pattern Sequence) e desempenho rebaixado na orelha esquerda em testes dicóticos com estímulo verbal, especificamente DD (dicótico de dígitos) e SSW (Staggered Spondaic Word $)^{(12-13)}$.

Já os prejuízos da integração provêm da inabilidade de integrar as informações sensoriais auditivas com outras não auditivas como visuais ou táteis. Ou seja, é o subperfil caracterizado pela dificuldade em tarefas que exijam transferência inter-hemisférica. Os achados típicos na avaliação comportamental do processamento auditivo são: déficit na nomeação de padrões temporais, com melhor performance com resposta do tipo imitação (PPS e DPS) e desempenho rebaixado na orelha esquerda em testes dicóticos com estímulo verbal (DD e SSW), principalmente em testes com maior complexidade linguística $(\mathrm{SSW})^{(10,14)}$.

O subperfil denominado associação auditiva caracterizase por dificuldades em reter a informação, acarretando baixa compreensão da leitura e de piadas, principalmente se forem apresentadas em voz passsiva. Na medida em que a demanda linguística aumenta, com a promoção para séries mais avançadas na escola, dificuldades podem se tornar mais aparentes $^{(8,10,14-15)}$. O achado típico na avaliação comportamental do processamento auditivo é o desempenho rebaixado nas duas orelhas em testes dicóticos com estímulo verbal (DD e SSW), sendo que frequentemente a orelha direita tem pior resultado.

Os comprometimentos relativos à organização de saída se referem à inabilidade em sequencializar, planejar e organizar respostas a uma informação ou a uma instrução de modalidade auditiva. Os achados típicos na avaliação comportamental do processamento auditivo caracterizam-se por apresentar baixo desempenho no teste de fala com ruído, em decorrência da dificuldade em habilidade de figura-fundo. Além disso, resultados rebaixados em testes que exijam o relato de vários elementos (respostas que devem ser dadas em ordem), como o SSW, o PPS e o DPS também podem ser encontrados ${ }^{(10,14)}$.

De acordo com os pressupostos estudados sobre processamento auditivo e desvio fonológico, torna-se possível refletir que um indivíduo com prejuízos na interpretação de infor- 
mações sensoriais por transtorno do processamento auditivo, poderá apresentar também, comprometimento na aquisição de seu sistema de linguagem. Dessa forma, a percepção e a produção da fala estão relacionadas e qualquer comprometimento na percepção da fala acometerá a produção da mesma ${ }^{(2)}$.

Um estudo com o objetivo de avaliar e analisar o desenvolvimento da linguagem em crianças cujas respostas auditivas comportamentais encontravam-se defasadas em relação à idade cronológica e apresentaram atraso na maturação auditiva, ou seja, concluiu que tais respostas podem ser um fator preditivo de desvio no desenvolvimento da linguagem ${ }^{(16)}$. Assim, o atraso no desenvolvimento linguístico foi maior nas crianças com um maior número de etapas defasadas na maturação auditiva.

Este trabalho tem como principal objetivo verificar as relações entre processamento auditivo e os desvios fonológicos por meio do estudo comparativo entre a ocorrência dos subperfis e dos desvios fonológicos, além dos testes de processamento auditivo e dos processos de substituição e estruturação silábica.

\section{MÉTODOS}

Esta pesquisa trata-se de um estudo observacional do tipo transversal, retrospectivo, no qual o fator em estudo é o desvio fonológico (variável independente) e o resultado dos testes e o subperfil do processamento auditivo constituem as variáveis dependentes.

A presente pesquisa foi aprovada pelo Comitê de Ética em Pesquisa do Centro Universitário Metodista IPA (protocolo $n^{\circ}$ $115 / 2007$ ) e devidamente autorizada pela instituição de origem. Não ofereceu riscos para saúde e integridade dos indivíduos e incluiu somente as crianças que tinham o termo consentimento para uso de informações assinado pelos responsáveis, autorizando os dados arquivados para pesquisa.

A pesquisadora realizou um levantamento, nos grupos de estágio de linguagem infantil, de pacientes com diagnóstico de desvio fonológico, que tivessem no mínimo sete anos de idade e que realizaram os testes de processamento auditivo nas clínicas integradas da instituição.

Nos prontuários, foram verificados os resultados da avaliação do processamento auditivo, da avaliação fonológica e informações sobre o gênero, a idade e série escolar. A coleta de dados foi realizada no período de agosto a setembro de 2007. Não foram incluídas crianças com síndromes, retardo mental ou crianças que apresentassem perda auditiva condutiva, neurossensorial ou mista. Para a avaliação fonológica, utilizou-se o instrumento Avaliação Fonológica da Criança ${ }^{(8)} \mathrm{e}$ a avaliação do processamento auditivo foi composta pela avaliação simplificada do processamento auditivo, PSI, Dicótico de Dígitos, SSW, PPS e DPS ${ }^{(17-20)}$.

A partir desse procedimento estratificou-se uma amostra de 15 crianças com faixa etária entre sete e 12 anos.

$\mathrm{Na}$ análise estatística foi utilizado o programa computacional The SAS (Statistical Analysis System), System for Windows versão 8.02 (SAS Institute Inc, 1999-2001, Cary, NC, USA).

Para descrever o perfil da amostra segundo as variáveis em estudo, foram realizadas tabelas de frequência das variáveis categóricas com valores de frequência absoluta (n) e percentual (\%), e estatísticas descritivas das variáveis contínuas como idade, escores dos testes de processamento auditivo, com valores de média, desvio padrão, valores mínimo e máximo, mediana e quartis.

Na comparação das variáveis categóricas entre os processos fonológicos foi utilizado o teste de Fisher.

Para comparar as variáveis numéricas entre os processos fonológicos com gênero, faixa etária e série escolar, utilizou-se o teste de Mann-Whitney, devido à ausência de distribuição normal das variáveis e ao tamanho reduzido da amostra. Para analisar a relação entre os escores dos testes de processamento auditivo e a idade das crianças foi utilizado o coeficiente de correlação de Spearman.

O nível de significância adotado para os testes estatísticos foi de $5 \%$, ou seja, $\mathrm{p}<0,05$.

\section{RESULTADOS}

Inicialmente serão descritas as variáveis: idade, sexo, série escolar, ocorrência dos processos fonológicos e subperfis de alteração do processamento auditivo.

A amostra foi constituída por quatro crianças com sete anos de idade (26,7\%), duas crianças com oito anos $(13,3 \%)$, duas crianças com nove anos (13,3\%); cinco crianças com 10 anos $(33,3 \%)$, uma criança com 11 anos $(6,7 \%)$ e uma criança com 12 anos $(6,7 \%)$, completados até a data da coleta de dados. A idade mínima da amostra foi sete anos, a máxima 12 anos e a média nove anos.

Em relação ao gênero, seis sujeitos eram meninas (40\%) e nove eram meninos $(60 \%)$.

Quanto à série escolar, cinco crianças cursavam a $1^{\mathrm{a}}$ série $(33,3 \%)$, quatro crianças cursavam a $2^{\mathrm{a}}$ série $(26,7 \%)$; duas crianças cursavam a $3^{\mathrm{a}}$ série $(13,3 \%)$, três crianças cursavam a $4^{\text {a }}$ série $(20 \%)$ e uma criança cursava a $5^{\text {a }}$ série $(6,7 \%)$ do ensino fundamental da rede estadual na data da coleta de dados.

Devido ao tamanho reduzido da amostra e à ocorrência de diversos processos fonológicos, utilizou-se a seguinte classificação: processos de substituição (para todos os processos em que há a substituição de um fonema por outro) e processos de estruturação silábica (para aqueles processos em que a estrutura da sílaba é alterada).

Assim, se obteve dois grupos: sete crianças apresentaram processos fonológicos de substituição $(46,7 \%)$, e outras oito crianças apresentaram processos fonológicos de substituição e estruturação silábica $(53,4 \%)$.

Em relação ao processamento auditivo todas as crianças (100\%) apresentaram pelo menos um subperfil alterado. Em relação a cada subperfil alterado, verificou-se que três crianças (20\%) tinham disfunções não específicas do processamento auditivo, ou seja, os resultados dos testes encontravam-se alterados em sua totalidade, não sendo representativos de nenhum dos cinco subperfis descritos; cinco crianças $(33,3 \%)$ tinham alteração do subperfil decodificação, duas crianças tinham alteração dos subperfis decodificação e integração $(13,3 \%)$; uma criança tinha alteração do subperfil integração $(6,7 \%)$, uma criança tinha alteração dos subperfis decodificação e associação $(6,7 \%)$, uma criança tinha alteração dos subperfis 
associação e função não-verbal (6,7\%); uma criança tinha alteração dos subperfis associação e organização de saída $(6,7 \%)$, uma criança tinha alteração dos subperfis decodificação, integração e função não-verbal (6,7\%). Para análise estatística os subperfis que tinham apenas um indivíduo foram reagrupados sob a denominação outros, devido à baixa frequência.

Na comparação das variáveis categóricas entre os processos fonológicos também houve agrupamentos quanto à idade (de sete a nove anos e de 10 a 12 anos) e quanto à série escolar (de $1^{\mathrm{a}}$ a $2^{\mathrm{a}}$ série e de $3^{\mathrm{a}}$ a $5^{\mathrm{a}}$ série).

Não houve diferença estatisticamente significante para gênero, idade, série escolar e subperfil de alteração do processamento auditivo ao comparar estas variáveis categóricas com os processos fonológicos de substituição e substituição e estruturação silábica.

Ao comparar os processos fonológicos de substituição e estruturação silábica aos resultados dos testes de processamento auditivo (variáveis numéricas) verificam-se diferenças estatisticamente significantes para a etapa de integração binaural orelha direita (IB OD) do teste dicótico de dígitos $(\mathrm{p}=0,018)$ e para a condição nomeando no teste PPS ( $\mathrm{p}=0,041)$. Tais achados significam que as crianças com processos de substituição e estruturação silábica apresentam maior alteração nos escores dos testes mencionados como mostra a Figura 1.

$\mathrm{Na}$ análise comparativa entre gênero e as principais variáveis categóricas (idade, série, subperfil) e numéricas (testes de processamento auditivo), não houve diferenças estatisticamente significantes.
Na comparação das faixas etárias com as variáveis categóricas (série escolar, e subperfis do processamento auditivo) não se obteve diferença estatisticamente significante. Já ao comparar as faixas etárias com os escores dos testes de processamento auditivo houve diferença estatisticamente significante no teste PSI OD para faixa etária de 10 a 12 anos $(\mathrm{p}=0,011)$, significando que o desempenho na realização do referido teste foi maior para essa faixa etária.

Quanto à análise comparativa entre as séries escolares e os subperfis do processamento auditivo não se obteve diferença estatisticamente significante. Já ao comparar a série escolar com o desempenho nos testes de processamento auditivo obteve-se diferença estatisticamente significante para aqueles que cursavam da $3^{\mathrm{a}}$ à $5^{\mathrm{a}}$ em relação aos que cursavam a $1^{\mathrm{a}} \mathrm{e}$ $2^{\mathrm{a}}$ série, sendo que os primeiros obtiveram escores maiores nos testes SSW na condição DC (direita competitiva) e etapa de ADD (atenção direcionada à direita) do teste DD. Esses resultados significam que o desempenho das crianças de $3^{\mathrm{a}}$ a $5^{\mathrm{a}}$ série nesses testes são superiores ao desempenho de crianças de séries anteriores, como pode ser observado na Figura 2.

$\mathrm{Na}$ análise de correlação entre idade e variáveis numéricas (testes de processamento auditivo) houve correlação estatisticamente significante para os testes PSI OD e DD ADD, sendo que as crianças mais velhas alcançaram os maiores escores. Assim, na medida em que a idade aumenta o desempenho nos referidos testes melhoram. Tais achados podem ser visualizados na Figura 3.
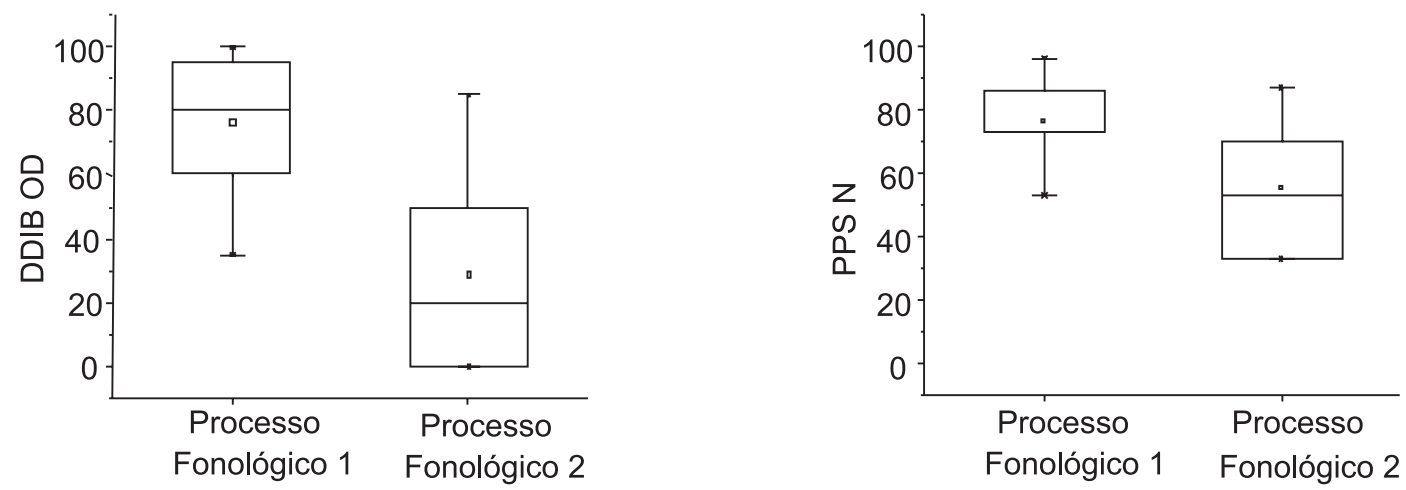

Figura 1. Resultados dos testes dicótico de dígitos (etapa integração binaural) e padrão sequencial de frequência - PPS (condição nomeando) na comparação entre processo de substituição (processo fonológico 1) e processo de substituição e de estruturação silábica (processo fonológico 2)
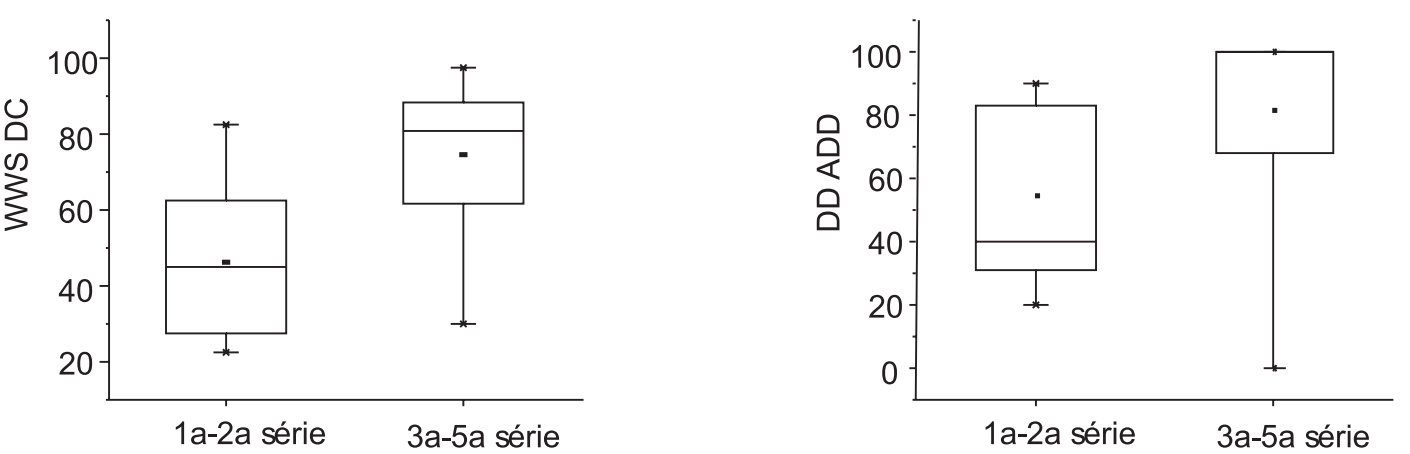

Figura 2. Resultado dos testes SSW (condição direita competitiva) e dicótico de dígitos (etapa de atenção direcionada a direita) na comparação entre as séries escolares 

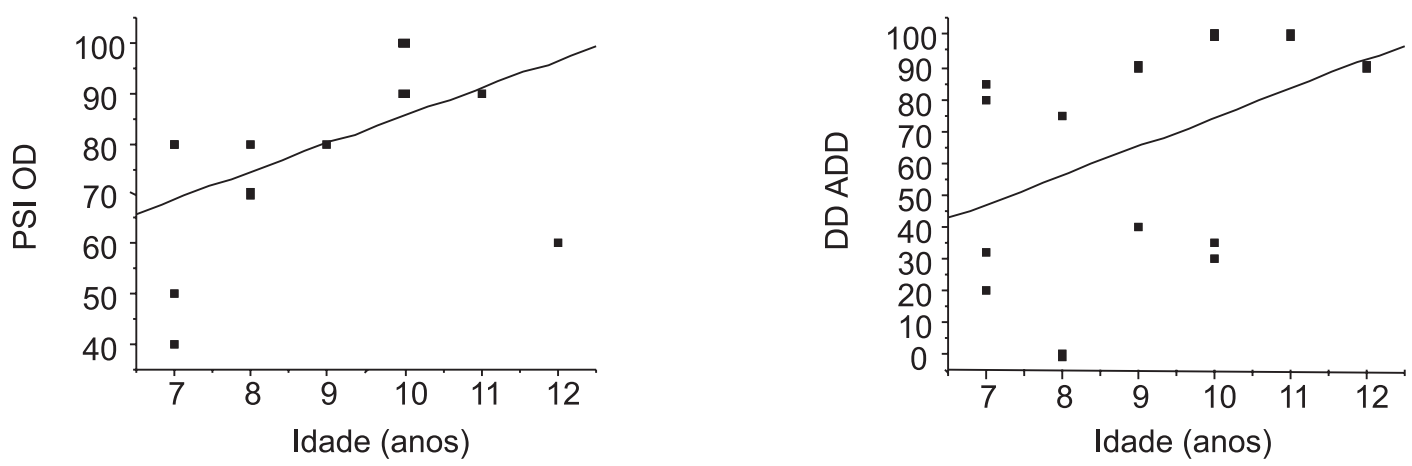

Figura 3. Crescimento dos testes PSI (orelha direita) e dicótico de dígitos (atenção direcionada a direita) em relação à idade

\section{DISCUSSÃO}

A faixa etária dos indivíduos da amostra varia entre sete e 12 anos sendo a média de nove anos de idade. A maturação do corpo caloso ocorre a partir dos sete anos de idade ${ }^{(12)}$ e se estende até 10 a 12 anos de idade ${ }^{(21-23)}$. Então, esses indivíduos ainda se encontram em processo maturacional dessa estrutura. Dentre os participantes da pesquisa $60 \%$ são do gênero masculino. Isso possivelmente se deve ao fato de meninos terem uma maior predisposição a alterações de linguagem. Em um estudo em que o critério de inclusão inicialmente era de crianças sem alterações do limiar de audibilidade e com queixas de alteração de fala foram encontrados 23 meninos e apenas três meninas que preenchiam esses critérios ${ }^{(24)}$.

Todas as crianças estudadas apresentaram transtorno do processamento auditivo, com pelo menos um subperfil alterado, sendo que o critério de inclusão era apenas ter realizado os testes de processamento auditivo, podendo apresentar ou não transtorno deste. Assim, os dados deste estudo sugerem a ocorrência concomitante do desvio fonológico e do transtorno do processamento auditivo.

Outros estudos também demonstraram uma tendência da simultaneidade de ocorrência de alterações na habilidade de processamento auditivo e de produção fonoarticulatória. Em um deles, a avaliação do processamento auditivo e a identificação das dificuldades de fala foram realizadas em 21 crianças de uma creche, com idade entre quatro anos e seis meses a cinco anos e 11 meses, observando que das 16 crianças com atraso ou distúrbio da produção fonoarticulatória, 13 apresentaram desvio ou desordem do processamento auditivo, demonstrando a importância da avaliação do processamento auditivo na presença de alterações fonoarticulatórias ${ }^{(6)}$.

$\mathrm{Na}$ presente pesquisa, foi verificada a maior ocorrência de alteração do subperfil decodificação $(33,3 \%)$, seguido de disfunções não específicas do processamento auditivo (20\%) e da alteração concomitante dos subperfis decodificação e integração $(13,3 \%)$.

Os resultados também demonstraram que as crianças que realizavam os dois processos fonológicos (substituição e estruturação silábica) em comparação àquelas que realizavam apenas processos de substituição, apresentaram maior alteração nos testes dicótico de dígitos para a etapa IBOD e PPS na condição nomeando. No primeiro, o rebaixamento da OD representa o desempenho das habilidades mediadas pelo córtex auditivo primário e secundário responsáveis pela retenção e discriminação dos padrões temporais, enquanto a alteração na condição nomeando do teste PPS remete ao funcionamento das habilidades inter-hemisféricas mediadas pelo corpo caloso ${ }^{(8,10,14)}$. Assim, as crianças cujos processos fonológicos interfiram na estrutura da sílaba apresentam mais transtornos de processamento auditivo.

Em um experimento foram avaliadas as habilidades auditivas de 25 crianças com prejuízo da produção fonoarticulatória, sendo 15 do sexo masculino e 10 do sexo feminino com faixa etária entre quatro anos e 11 meses e oito anos de idade. Essas crianças foram reunidas em dois grupos: seis crianças fizeram parte do grupo de alterações articulatórias superficiais, cuja, a manifestação principal era dessonorização; e as outras 19 crianças constituíam outro grupo com mais alterações no sistema fonêmico. Dentre todas as crianças da pesquisa $72 \%$ apresentaram distúrbio do processamento auditivo, sendo $33,33 \%$ do primeiro grupo e $84,21 \%$ do segundo grupo. Esse estudo evidenciou que dificuldades de produção fonoarticulatórias envolvendo vários fonemas podem estar associadas a habilidades auditivas prejudicadas ${ }^{(6)}$.

Ao comparar as faixas etárias com os escores dos testes de processamento auditivo, houve diferença estatisticamente significante no teste PSI OD para faixa etária de 10 a 12 anos. As habilidades envolvidas neste teste são figura-fundo e atenção seletiva. Este achado significa que o desempenho na realização do referido teste foi maior para essa faixa etária. Assim concorda-se com outros estudos que observaram a melhora no desempenho nos testes de processamento auditivo com o aumento da idade ${ }^{(13,25-27)}$.

O mesmo ocorreu com as séries escolares, em que foi observado que quanto mais avançado o grau de instrução melhor o desempenho nos testes SSW DC e DD ADD do processamento auditivo.

Ao relacionar estes achados com a teoria vigente sobre os desvios fonológicos constata-se que a ocorrência de processos fonológicos diminui conforme a idade aumenta, pois ocorre um amadurecimento do conhecimento fonológico no decorrer do tempo ${ }^{(6)}$. O mesmo acontece no desempenho nos testes de processamento auditivo que também melhoram com a idade ${ }^{(13,25-27)}$.

Esta pesquisa confirmou a relação entre processamento auditivo e desvio fonológico, mesmo com uma amostra pequena $(n=15)$. Certamente, outras pesquisas, com um número maior 
de participantes, buscando fazer essa relação, demonstrariam resultados ainda mais surpreendentes.

Um estudo realizado a partir da análise das características de fala e da percepção auditiva de 20 crianças, com idade entre quatro e seis anos, que apresentavam o processo fonológico denominado ensurdecimento (dessonorização) entre outros, evidenciou a necessidade de avaliação da percepção auditiva já que $70 \%$ dessas crianças falharam em pelo menos uma das provas da triagem do processamento auditivo, a maioria em memória sequencial verbal $(65 \%)^{(28)}$.

Segundo a American Speech Hearing Language Association (ASHA), existe uma relação causal entre problemas de linguagem e distúrbio do processamento auditivo, sobretudo em nível da compreensão da linguagem oral ${ }^{(29)}$.

O desenvolvimento da fala é influenciado pelas capacidades perceptivas da criança ${ }^{(30)}$. É por meio da recepção, da análise e da organização do processamento das informações auditivas que se estabelece a representação mental do estímulo linguístico e o armazenamento dessa representação na memória ${ }^{(28)}$, que envolve o processamento temporal. Este estudo não incluiu testes mais específicos para a avaliação desta habilidade. Assim, mais pesquisas deverão ser realizadas para investigar o processamento temporal em crianças com desvio fonológico.

\section{CONCLUSÃO}

Apesar do tamanho amostral pequeno, os dados desta pesquisa apontam para a construção da relação entre processamento auditivo e desvio fonológico e demonstram que as crianças que realizaram processos fonológicos que interferiram na estrutura da sílaba, além dos processos de substituição, apresentaram mais alterações nos testes de processamento auditivo do que aquelas que realizaram somente processos de substituição.

Dessa forma, outras pesquisas com um número maior de participantes devem ser realizadas objetivando a correlação de ambas as variáveis.

\begin{abstract}
Purpose: To study the relationship between phonological disorder and auditory processing. Methods: Data were gathered from patients' records, and included individuals with diagnosis of phonological disorder, with seven years old or more, who had carried out auditory processing tests. The study considered auditory processing evaluation, speech evaluation, gender, age, and school grade. Results: All subjects (100\%) presented at least one subprofile of the auditory processing altered. When compared the presence of substitution and syllabic structure processes to the results of auditory processing tests, a statistically significant correlation was found for the stage of binaural integration in the right ear on the dichotic digits test $(\mathrm{p}=0.018)$, and for the naming condition of the PPS test $(\mathrm{p}=0.041)$. When compared the auditory processing tests to the age variable, there was a statistically significant difference in the PSI test in the right ear $(\mathrm{p}=0.011)$ for the age range from ten to 12 years. The same result was found in the comparison between auditory processing tests and school grade, in which the right competitive condition of the SSW test $(\mathrm{p}=0.039)$ and attention directed to the right on the dichotic digits test $(\mathrm{p}=0.037)$ had statistically significant results for the more advanced school grades. Conclusions: This study suggested the existence of a close relationship between auditory processing and phonological disorder, especially regarding the performance of the right ear. This finding evidences the importance of investigating the occurrence of deficits in the auditory abilities of children with phonological disorder.
\end{abstract}

Keywords: Hearing; Auditory perception; Developmental disabilities; Language; Speech disorders

\section{REFERÊNCIAS}

1. Mota HB, Keske-Soares M, Bagetti T, Ceron MI, Filha MGCM. Análise comparativa de três diferentes modelos de terapia fonológica. Pró-Fono. 2007;19(1):67-74.

2. Raitano NA, Pennington BF, Tunick RA, Boada R, Shriberg LD. Preliteracy skills of subgroups of children with speech sound disorders. J Child Psychol Psychiatry. 2004;45(4):821-35.

3. Lamprecht RR. Sobre os desvios fonológicos. In: Lamprecht RR, organizador. Aquisição fonológica do português: perfil de desenvolvimento e subsídios para terapia. Porto Alegre: Artmed; 2004. p. 193-212.

4. Yavas M, Hernandorena CLM, Lamprecht RR. Avaliação fonológica da criança: reeducação e terapia. Porto Alegre: Artes Médicas; 1992.

5. Oliveira MMF, Wertzner HF. Estudo do distúrbio fonológico em crianças. Rev Soc Bras Fonoaudiol. 2000;7(1):68-75.

6. Pereira LD, Ortiz KZ. Desordem do processamento auditivo central e distúrbios da produção fonoarticulatória. In: Lichtig I, Carvalho RMM, organizadores. Audição: abordagens atuais. São Paulo: Pró-Fono; 1997. p. $173-86$.
7. Abraham SS, Wallace IF, Gravel JS. Early otitis media and phonological development at age 2 years. Laryngoscope. 1996;106(6):727-32.

8. Costa-Ferreira MID. A influência da terapia do processamento auditivo na compreensão em leitura: uma abordagem conexionista. [tese]. Porto Alegre: Faculdade de Letras da Pontifícia Universidade Católica do Rio Grande do Sul; 2007.

9. Corona AP, Pereira LD, Ferrite S, Rossi AG. Memória sequencial de três e quatro sílabas em escolares. Pró-Fono. 2005;17(1):27-36.

10. Bellis TJ. Assessment and management of central auditory processing disorders in the educational setting: from science to practice. 2a ed. New York: Thomson Delmar Learning; 2003.

11. Alvarez AMMA, Caetano AL, Nastas SS. Processamento auditivo central: avaliação e diagnóstico. Fono Atual. 1997;1(1):346.

12. Alvarez AMMA, Zaidan E, Balen SA, Garcia AP. Disfunção não verbal. Acta AWHO. 2000;19(1):49-55.

13. Schochat E, Rabelo CM, Sanfins MD. Processamento auditivo central: 
testes tonais de padrão de frequência e de duração em indivíduos normais de 7 a 16 anos de idade. Pró-Fono. 2000;12(2):1-7.

14. Alvarez AMMA, Balen AS, Misoreli MIL, Sanchez, ML. Processamento auditivo central: proposta de avaliação e diagnóstico diferencial. In: Munhoz MSL, Caovilla HH, Silva MLG, Ganança MM, editores. Audiologia clínica. São Paulo: Atheneu; 2000. [Série otoneurológica]

15. Costa-Ferreira MID, Mello AM. Comorbidade entre transtorno de déficit de atenção e hiperatividade e distúrbio do processamento auditivo. Fonoaudiol Brasil. 2006;4(1):5.

16. Kaminski JM, Tochetto TM, Mota HB. Maturação da função auditiva e desenvolvimento de linguagem. Rev Soc Bras Fonoaudiol. 2006;11(1):17-21.

17. Ziliotto KN, Kalil DM, Almeida CIR. PSI em português. In: Pereira LD, Schochat E. Processamento auditivo central: manual de avaliação. São Paulo: Lovise; 1997. p.113-28.

18. Santos MFC, Pereira LD. Escuta com dígitos. In: Pereira LD, Schochat E. Processamento auditivo central: manual de avaliação. São Paulo: Lovise; 1997. p.147-50.

19. Borges ACLC. Dissílabos alternados - SSW. In: Pereira LD, Schochat E. Processamento auditivo central: manual de avaliação. São Paulo: Lovise; 1997. p.169-78.

20. Auditec. Evaluation manual of pitch pattern sequence and duration pattern sequence. Missouri, USA: Auditec; 1997.

21. Kraus N. Auditory pathway encoding and neural plasticity in children with learning problems. Audiol Neurootol. 2001;6(4): 221-7.
22. Ponton CW, Eggermont JJ, Kwong B, Don M. Maturation of human central auditory system activity: evidence from multi-channel evoked potentials. Clin Neurophysiol. 2000;111(2):220-36.

23. Purdy SC, Kelly AS, Darvies MG. Auditory brainstein response, middle latency response, and late cortical evoked potentials in children with learning disabilities. J Am Acad Audiol. 2002;13(7):367-82.

24. Fernandes JB, Toniolo IMF, Cechella C. Habilidades auditivas centrais em meninos com desvio fonológico. Fonoaudiol Brasil. 2000;3(3):1523.

25. Chermak GD, Musiek FE. Managing central auditory processing disorders in children and youth. Am J Audiol. 1992;1(3):61-5.

26. Phillips DP. Central auditory system and central auditory processing disorders. Semin Hear. 2002;23(4):251-61.

27. Costa LP, Pereira LD, Santos MFC. Auditory fusion test in scholars. Pró-Fono. 2004;16(2):187-96.

28. Magalhães ATM, Paolucci JF, Ávila CRB. Estudo fonológico e da percepção auditiva de crianças com ensurdecimento de consoantes. Fono Atual. 2006;8(35):22-9.

29. Task Force on Central Auditory Processing Consensus Development. Central Auditory Processing: current status of research and implications for clinical practice. Am J Audiol. 1996;5(2):41-52.

30. Pagan LO, Wertzner HF. Análise acústica das consoantes líquidas do Português Brasileiro em crianças com e sem transtorno fonológico. Rev Soc Bras Fonoaudiol. 2007;12(2):106-13. 